\title{
Finite utility on financial markets with asymmetric information and structure properties of the price dynamics
}

\author{
Stefan Ankirchner, Peter Imkeller* \\ Institut für Mathematik, Humboldt-Universität zu Berlin, Unter den Linden 6, 10099 Berlin, Germany
}

Received 20 November 2003; received in revised form 9 February 2004; accepted 2 March 2004

Available online 25 March 2005

\begin{abstract}
We consider financial markets with two kinds of small traders: regular traders who perceive the (continuous) asset price process $S$ through its natural filtration, and insiders who possess some information advantage which makes the filtrations through which they experience the evolution of the market richer. We discuss the link between (NFLVR), the semimartingale property of $S$ viewed from the agent's perspective, and bounded expected utility. We show that whenever an agent's expected utility is finite, $S$ is a semimartingale with a Doob-Meyer decomposition featuring a martingale part and an information drift. The expected utility gain of an insider with respect to a regular trader is calculated in a completely general setting. In particular, for the logarithmic utility function, utility gain is a function of the relative information drift alone, regardless of whether the market admits arbitrage. (C) 2005 Elsevier SAS. All rights reserved.
\end{abstract}

\section{Résumé}

On considère un marché financier avec deux sortes de petits investisseurs : des investisseurs réguliers qui perçoivent l'évolution du prix $S$ dans sa filtration naturelle, et des agents initiés ayant des informations supplémentaires et qui ainsi suivent l'évolution du prix par une filtration plus riche. On discute le rapport entre (NFLVR), la propriété de semimartingale de $S$ vue dans la perspective de l'agent, et la bornitude de l'espérance de l'utilité. Si celle-ci est bornée, $S$ est une semimartingale avec une décomposition de Doob-Meyer comprenant une martingale et un drift d'information. On calcule l'utilité supplémentaire espérée pour l'agent initié sous des conditions générales. On montre, que si la fonction d'utilité est logarithmique, l'utilité supplémentaire ne dépend que du drift d'information. C'est le cas même si le marché admet un arbitrage.

() 2005 Elsevier SAS. All rights reserved.

MSC: primary 60G48, 60H05, 91B28; secondary 93E20, 94A17, 60H30

Keywords: Insider trading; Enlargement of filtration; Free lunch with vanishing risk; (NFLVR); Arbitrage; Finite expected utility;

Semimartingale; Stochastic integrator; Information drift

\footnotetext{
This work was partially supported by the DFG research center 'Mathematics for key technologies' Matheon in Berlin.

* Corresponding author.

E-mail address: imkeller@mathematik.hu-berlin.de (P. Imkeller).
} 


\section{Introduction}

Asymmetry of information on financial markets has been a subject of increasing interest in recent years. Several mathematical models have been designed to deal with financial markets on which traders with different information levels are active. See Wu [32] for an overview. The model to capture basic facts of insider's action on markets which motivated this paper is very simple. Two kinds of traders are considered: regular agents who do not know any more than the natural evolution of the assets of the market, and insiders whose knowledge at any given time in the trading interval is larger than the $\sigma$-field generated by the asset price process up to that time. The insider may, for example, possess some additional information on the price of an asset at maturity, or at some later time. He might anticipate the time when an asset price reaches a favorable level, or be able to stop at the time at which some final level crossing of the price process occurs. Situations of this type have been modelled for example by Karatzas, Pikovsky [22], Amendinger [1], Amendinger, Becherer and Schweizer [2], Grorud, Pontier [16], and [3,19,17,18]. In most of these papers, questions of utility gain of the insider relative to the regular trader were discussed. It turned out that for many types of additional information the expected increment of utility gained by the insider may become infinite quite easily, and might provide opportunities for free lunch or even arbitrage in an equally easy way. Baudoin [5,6] and Baudoin, Nguyen-Ngoc [7] develop a model in which additional information on some random variable unknown to the regular trader is only weakly available, i.e. in the form of some knowledge of its law instead of the precise anticipation of its value. In this framework the insider's utility is more likely to be finite and can be computed for example by means of the fundamental results by Kramkov, Schachermayer [23]. In [10], the precise observation of some random element by the insider which is inaccessible to the natural trader is blurred dynamically by some exterior independent noise to produce a weaker information advantage in the same spirit, and keep the additional utility from getting out of control.

A natural mathematical toolbox to use in the context of the models described contains the techniques of grossissement de filtrations developed in some deep work mostly by French authors [9,20,21,25,33-36,31]. This is just one of numerous examples in which the direct impact of Meyer's Strasbourg school on contemporary financial mathematics becomes evident. Another example is initiated in a recent paper by Biagini and Oksendal [8]. In this paper a question is raised which appears to be of purely mathematical interest at first glance: knowing that the expected utility of an insider is finite, what can be said about the regularity of the asset price process from the insider's point of view? The authors show that given finite utility and the existence of an optimal investment strategy for the insider, the asset price process must be a semimartingale in the insider's enlarged filtration. This way, they address one of the basic questions of the theory of grossissements de filtrations, and at the same time raise a problem which goes to the heart of stochastic analysis: the relationship between semimartingales and the stochastic integrator property. To describe the utility of the insider in his enlarged filtration, they use extended notions of stochastic integrals investigated in anticipative stochastic calculus, such as Skorokhod's integral (see Nualart [26]) and the forward Itô integral introduced by Russo and Vallois [29].

The deep and central theorem of Bichteler-Dellacherie-Mokobodski characterizes semimartingales as good stochastic integrators. A process $S$ is a semimartingale if and only if the stochastic integrals of uniformly bounded simple processes, i.e. predictable step processes, with respect to $S$ form a bounded set in the topological vector space of random variables with the $\left(L^{0}\right.$-)topology of convergence in probability. This key theorem allows to deal with the problem posed by Biagini and Oksendal [8] from a different perspective. Suppose an agent invests on a financial market with asset price process $S$ and measures the utility of his final wealth through a utility function $U$ which is unbounded. Then the hypothesis that the expected utilities the agent is able to attain be a bounded function of the simple investment strategies he is allowed to use due to his information horizon should be closely related to the $L^{0}$-boundedness of the set of stochastic integrals of simple admissible strategies. Hence the theorem should indicate a direct link between finite utility of agents on financial markets and the semimartingale property of the asset price processes with respect to the evolution of their information. This basic observation is the starting point for the analysis presented in this paper. 
A related link is exploited in the fundamental paper by Delbaen and Schachermayer [11]. It is shown that if an asset price process $S$ fulfills the (NFLVR) condition, i.e. allows no admissible simple strategies which lead with positive probability to a final gain with controllable risk, then the agent views $S$ as a semimartingale. In addition, $M$ being the martingale part of $S$, its Doob-Meyer decomposition is given by the special formula

$$
S=M+\alpha \cdot\langle M, M\rangle .
$$

We start by proving that if an agent has bounded expected utility with respect to his information horizon, then he cannot have (FLVR). This allows us to hook up to the result by Delbaen and Schachermayer, to show that bounded utility implies the semimartingale property of $S$, and to investigate more thoroughly the relationship between the properties: (NFLVR), the semimartingale property of $S$ in the agent's filtration, and bounded expected utility. The drift density $\alpha$ may be considered as a function of the agent's information horizon, i.e. its filtration. Passing from one filtration to a bigger one while keeping utility finite will change $\alpha$ to $\beta$, and we may well call $\beta-\alpha$ the corresponding information drift. We will keep an attentive eye on logarithmic utility. In this particular case we will show that a better informed agent's additional utility is a function of the information drift alone, regardless of whether we face a complete or an incomplete market. This result is derived in an entirely abstract framework. We do not have to specify the type of information advantage the insider possesses. Based on the fundamental result by Kramkov and Schachermayer [23], we will describe the additional expected utility of an insider in a complete market setting for all reasonable utility functions and express it as a function of relative information drifts.

Here is a brief outline of the paper. In Section 1, we shall investigate the relationship between (NFLVR), the semimartingale property, and finite utility. In Section 2 we restrict our attention to logarithmic utility. We calculate it in general incomplete market settings, and derive the expected utility increment of better informed agents as a function of the universal information drift of his filtration. In Section 3 we transfer these results to the setting of more general utility functions in complete markets, and show that the logarithm gives essentially the only way of measuring utility which allows portfolios that are optimal at any time in the trading interval.

\subsection{Preliminaries and notation}

Here we collect the most important definitions, notations and conventions needed throughout the paper. Let $(\Omega, F, P)$ be a probability space and $\mathcal{F}=\left(\mathcal{F}_{t}\right)_{0 \leqslant t \leqslant T}$ an arbitrary filtration satisfying the usual conditions, where $T$ is the finite time horizon. Suppose that $S:[0, T] \times \Omega \rightarrow \mathbb{R}$ is a stochastic process. $S$ will take the role of the asset price process on our financial market. The wealth of the agent on our market with information horizon $\mathcal{F}$ will be determined in the subsequent section by simple investment strategies (integrands) of the following form.

Definition 0.1. A simple integrand is a linear combination of processes of the form $f 1_{\left.] T_{1}, T_{2}\right]}$ where $f$ is a bounded and $\mathcal{F}_{T_{1}}$-measurable random variable and $T_{1}$ and $T_{2}$ are stopping times with values in $[0, T]$ and with respect to the filtration $\mathcal{F}$.

The collection of simple integrands will be denoted by $\mathcal{S}$ and the stochastic integral process of simple or more general predictable integrands with respect to a cadlag process $X$ by $\theta \cdot X$. We now recall some terminology introduced in [11]. If $a$ is a positive real number, then a strategy $\theta$ is called $a$-admissible, if for all $t \in[0, T]$ we have $(\theta \cdot S)_{t} \geqslant-a$ almost surely. It will be called admissible if it is $a$-admissible for some $a \geqslant 0$. We put

$$
\mathcal{K}^{s}=\left\{(\theta \cdot S)_{T} \mid \theta \in \mathcal{S} \text { admissible }\right\}
$$

and write $C^{s}$ for the set of functions dominated by elements of $\mathcal{K}^{s}$, i.e. $C^{s}=\mathcal{K}^{s}-L_{+}^{0}$. Now let $C=C^{s} \cap L^{\infty}$. The process $S$ is said to satisfy the no free lunch with vanishing risk (NFLVR) property for simple integrands, if

$$
\bar{C} \cap L_{+}^{\infty}=\{0\},
$$


where $\bar{C}$ denotes the closure of $C$ in $L^{\infty}$. If the intersection contains more than the trivial random variable 0 , we shall say that $S$ satisfies (FLVR) for simple integrands. For the general (NFLVR) condition we refer to $\mathcal{K}$ defined as $\mathcal{K}^{s}$ just with general $\mathcal{F}$-predictable $\theta$ with well defined stochastic integral.

If not stated otherwise in the sequel, we mean by a utility function a function $U: \mathbb{R} \rightarrow[-\infty, \infty)$ which is strictly concave and strictly increasing on $\operatorname{dom}(U)=\{y: U(y)>-\infty\}$. We will interpret the integral $E U\left(x+(\theta \cdot S)_{T}\right)$ as the expected utility from terminal wealth of a trader possessing an initial wealth $x$ and choosing his investments following the strategy $\theta$. Note that the integral might not exist. For ease of notation, we use the convention $E U(x+$ $\left.(\theta \cdot S)_{T}\right)=-\infty$, if both the positive and the negative parts of $U\left(x+(\theta \cdot S)_{T}\right)$ have infinite expectations.

\section{Finite utility and semimartingales}

The analysis of this paper is strongly motivated by [8]. With the aim of calculating the expected utility increment of a better informed agent on a financial market, one of the main topics of [8] consists in showing that boundedness of (logarithmic) utility implies the semimartingale property of the price process $S$ viewed from the perspective of the better informed agent. His additional information is mirrored by his filtration $\mathcal{G}$, the natural evolution of information $\mathcal{F}$ with respect to which $S$ is adapted, initially enlarged by some extra random element. To reach this goal, the authors argue as in other papers (see for example Leon et al. [24]) via anticipative calculus embedded in Malliavin's calculus in an extrinsic approach. They view the better informed agent's world from the natural evolution of information perspective as anticipative, therefore work with an extended version of Itô's calculus which needs conversion formulas between Itô's and Skorokhod's integrals given by Malliavin trace terms by means of which the additional expected utility of better informed agents is ultimately computed. The authors consequently are forced to restrict their studies to particular stochastic bases such as Wiener spaces, and in addition require unnecessary assumptions concerning regularity in terms of the stochastic calculus of variations.

In contrast to this approach, we propose to look at the problem from an intrinsic point of view. For any agent, fixing the filtration describing his particular view of information flow, irrespective of other agents' filtrations, we propose to compute the maximal expected utility for rather general utility functions with respect to the agent's basis, employing the powerful tools of general semimartingale theory already exploited in several deep papers by Delbaen and Schachermayer. Of course, thereby the semimartingale property of $S$ in the general agent's filtration has to play a decisive role. It is tackled by a key observation made in Delbaen and Schachermayer [11] establishing a link between the (NFLVR) condition and the semimartingale property of an asset price process on a financial market. So, to find a natural answer to the problem discussed in [8] in a general and natural framework, we have to complete the study of the relationships between (NFLVR) and the semimartingale property by linking both of these properties to a third one: finiteness of expected maximal utility. This is the task of the present section. In fact, the main work load needed thereby is already taken by [11], and we may consider the modest contribution of our paper in finding the shortcut on the route of arguments used in [8] and other papers through (NFLVR). Only after doing this, in Sections 2 and 3 we propose to compare the obtained optimal expected utilities starting with the logarithmic one, and work out the increment of the better informed agent in terms of a universal quantity which we may call information drift.

Throughout this section, we let $S$ have cadlag paths and be adapted to $\mathcal{F}$. Following our intention to consider the optimal utility increment of a better informed agent from an intrinsic point of view, we interpret $\mathcal{F}$ to be any agent's information horizon. Only later we shall distinguish different filtrations. For the moment we do not need any more assumptions. Only in the end of the Subsection 1.1 we shall sometimes assume local boundedness of $S$.

\subsection{Finite utility via simple strategies}

In this subsection we explore the relationship between finiteness of utility and the semimartingale property of the price dynamics allowing only finite combinations of buy-and-hold strategies, i.e. strategies taken from $\mathcal{S}$. 
The following is a useful reformulation of the (FLVR) property.

Lemma 1.1. $S$ satisfies the (FLVR) property for simple integrands (see Definition 0.1) if and only if there is a sequence $\left(\theta^{n}\right)_{n \geqslant 0}$ of admissible simple integrands such that the following two conditions are satisfied

(i) $f_{n}=\left(\theta^{n} \cdot S\right)_{T}, n \in \mathbb{N}$, converges a.s. to a nonnegative function $f$ satisfying $P(f>0)>0$ and

(ii) $\left\|f_{n}^{-}\right\|_{\infty} \rightarrow 0$.

Proof. This follows from Proposition 3.6 in [11]. Although the statement there is shown for general integrands the result pertains if only simple strategies are considered.

The following proposition provides the link between the boundedness of the agent's utility for simple strategies and the (NFLVR) condition.

Proposition 1.2. Let $U: \mathbb{R} \rightarrow[-\infty, \infty)$ be a utility function with $\lim _{x \rightarrow \infty} U(x)=\infty$. Then for all $x>$ $\sup \{y \in \mathbb{R}: U(y)=-\infty\}$ (recall $\sup \emptyset=-\infty)$ the following implication holds.

If $\sup _{\mathcal{S} \ni \theta \text { admissible }} E\left[U\left(x+(\theta \cdot S)_{T}\right)\right]<\infty, \quad$ then $(N F L V R)$ for simple integrands.

Proof. Let $x>\sup \{y \in \mathbb{R}: U(y)=-\infty\}$. Then there is a $\delta>0$ for which $x-\delta>\sup \{y \in \mathbb{R}: U(y)=-\infty\}$. We put $D=U(x-\delta) \wedge 0>-\infty$.

Suppose that the (NFLVR) property for simple integrands is violated. By the preceding lemma we can find a sequence $\left(\theta^{n}\right)_{n \in \mathbb{N}}$ of admissible simple integrands such that the final payoffs $f_{n}=\left(\theta^{n} \cdot S\right)_{T}, n \in \mathbb{N}$, satisfy

(i) $f_{n}=\left(\theta^{n} \cdot S\right)_{T} \rightarrow f$ a.s., where $f$ is nonnegative with $P(f>0)>0$ and

(ii) $\left\|f_{n}^{-}\right\|_{\infty} \rightarrow 0$.

For $n \in \mathbb{N}$ we set $\varepsilon_{n}=\left\|f_{n}^{-}\right\|_{\infty}$. For all but finitely many $n \in \mathbb{N}$ we have $\varepsilon_{n}<\delta$. To simplify notation we assume that this holds for all $n \in \mathbb{N}$. We now define new simple integrands

$$
\pi^{n}=\frac{\delta}{\varepsilon_{n}} \theta^{n}
$$

for all $n \in \mathbb{N}$. It is clear that all the integrals $\left(\pi^{n} \cdot S\right)_{T}$ exceed the bound $-\delta$. Furthermore the random variables $U\left(x+\left(\pi^{n} \cdot S\right)_{T}\right)$ are bounded from below by the constant $D$. More formally,

$$
U\left(x+\left(\pi^{n} \cdot S\right)_{T}\right)=U\left(x+\frac{\delta}{\varepsilon_{n}}\left(\theta^{n} \cdot S\right)_{T}\right) \geqslant U\left(x+\frac{\delta}{\varepsilon_{n}}\left(-\varepsilon_{n}\right)\right)=U(x-\delta)=D>-\infty .
$$

Since $f_{n}$ converges to the nontrivial nonnegative function $f$, one can find an integer $n_{0}$ and real numbers $\alpha, c>0$ such that

$$
P\left(\left(\theta^{n} \cdot S\right)_{T}>b\right)>c
$$

for all $n \geqslant n_{0}$. Recalling that $D \leqslant 0$, this is seen to imply

$$
\begin{aligned}
\liminf _{n \rightarrow \infty} E\left[U\left(x+\left(\pi^{n} \cdot S\right)_{T}\right)\right] & =\liminf _{n \rightarrow \infty} E\left[U\left(x+\frac{\delta}{\varepsilon_{n}}\left(\theta^{n} \cdot S\right)_{T}\right)\right] \\
& \geqslant \liminf _{n \rightarrow \infty} E\left[D 1_{\left\{\left(\theta^{n} \cdot S\right)_{T} \leqslant b\right\}}+U\left(x+\frac{\delta}{\varepsilon_{n}} b\right) 1_{\left\{\left(\theta^{n} \cdot S\right)_{T}>b\right\}}\right] \\
& \geqslant \liminf _{n \rightarrow \infty}\left[D(1-c)+U\left(x+\frac{\delta}{\varepsilon_{n}} b\right) c\right]=\infty .
\end{aligned}
$$


Hence

$$
\sup _{\mathcal{S} \ni \theta \text { admissible }} E\left[U\left(x+(\theta \cdot S)_{T}\right)\right]=\infty .
$$

This proves the proposition.

Remark. Proposition 1.2 holds in particular for all increasing functions $U$ with $\lim _{x \rightarrow \infty} U(x)=\infty$.

Combining Proposition 1.2 with the results of the fundamental paper by Delbaen and Schachermayer [11] we obtain the intuitively plausible relationship between boundedness of the expected utility and the semimartingale property for the continuous asset price process with respect to the agent's filtration. Note that this already contains the natural generalization of the answer to the first main question in [8].

Corollary 1.3. Let $S$ be locally bounded, $U: \mathbb{R} \rightarrow[-\infty, \infty)$ a utility function with $\lim _{x \rightarrow \infty} U(x)=\infty$ and $x>\sup \{y \in \mathbb{R}: U(y)=-\infty\}$. If $\sup _{\mathcal{S} \ni \theta \text { adm. }} E\left[U\left(x+(\theta \cdot S)_{T}\right)\right]<\infty$, then $S$ is a semimartingale with respect to $\mathcal{F}$.

Proof. By Proposition 1.2, the process $S$ satisfies the (NFLVR) property for simple integrands. Theorem 7.2 in Delbaen and Schachermayer [11] states that in this case $S$ is already a semimartingale. Note that Delbaen and Schachermayer [11] use a slightly different definition of simple integrands than stated in Definition 0.1. They allow for unbounded processes. But one can show that (NFLVR) for bounded simple integrands is equivalent to (NFLVR) for all (possibly unbounded) simple processes.

Remark. We can sharpen the result of the preceding corollary. In fact, we can show that boundedness of expected utility over all simple strategies uniformly bounded by some constant is sufficient for the semimartingale property of $S$ to hold. For the statement and proof of this property we refer to the thesis by Ankirchner [4], and a forthcoming paper.

\subsection{Simple versus general strategies}

In the preceding subsection we have seen that if the expected utility maximized over the set of simple strategies is finite, the price process $S$ is a semimartingale. As a consequence, $S$ is a stochastic integrator, and its stochastic integral is defined not only for simple integrands, but for a much wider class of $\mathcal{F}$-predictable strategies. A natural question arising in this context is the following: can a trader increase his optimal utility by using general $S$-integrable strategies? While this may be the case for discontinuous $S$, as is shown by an example at the end of this subsection, its main result will prove that for continuous asset price processes $S$ the answer is no.

The utility functions $U: \mathbb{R} \rightarrow[-\infty, \infty)$ considered in this section have to fulfill the following further requirements. We suppose that $U$ is strictly increasing, strictly concave and continuously differentiable on $\operatorname{dom}(U)=$ $\{y: U(y)>-\infty\}$. Furthermore we assume that the Inada conditions are satisfied, i.e.

$$
U^{\prime}(\infty)=\lim _{x \rightarrow \infty} U^{\prime}(x)=0
$$

and

$$
U^{\prime}(c)=\lim _{x \downarrow c} U^{\prime}(x)=-\infty,
$$

where $c=\inf \{y: U(y)>-\infty\} \in[-\infty, \infty)$. 
We remark at this point that all results of this section could equally well be stated for infinite time horizon. For homogeneity reasons (some results we refer to in a later section are formulated for finite $T$ ) we refrain from doing so.

Throughout this section we suppose that $S$ is an $\mathcal{F}$-semimartingale and we denote by $\mathcal{A}$ the set of all $\mathcal{F}$-predictable processes $\theta$ which are integrable with respect to $S$ in the sense of Protter (see Section 2, Chapter IV in [27]). As in the previous subsection we use for all $\theta \in \mathcal{A}$ the convention $E\left[U\left(x+(\theta \cdot S)_{T}\right)\right]=-\infty$, if both the negative and the positive parts are not integrable.

We next define two quantities to be compared to the maximal expected utility taken over simple strategies. Fix an initial wealth $x>\sup \{y: U(y)=-\infty\}$. Let

$$
u_{a}(x)=\sup _{\mathcal{A} \ni \theta \text {-adm. }} E\left[U\left(x+(\theta \cdot S)_{T}\right)\right]
$$

and

$$
u(x)=\sup _{\mathcal{A} \ni \theta \text { adm. }} E\left[U\left(x+(\theta \cdot S)_{T}\right)\right]
$$

Before stating the main result of this subsection, some preliminary steps are in order. The following auxiliary results deal with some aspects of $a$-admissible strategies.

Lemma 1.4. Let $S$ be a continuous semimartingale satisfying (NFLVR). If $\theta$ is a-admissible then almost surely

$$
(\theta \cdot S)_{T}>-a \Longrightarrow(\theta \cdot S)_{t}>-a \text { for all } 0 \leqslant t \leqslant T \text {. }
$$

Proof. Let $A=\left\{\right.$ there exists a $t \in[0, T]$ for which $\left.(\theta \cdot S)_{t}=-a\right\}$. A is measurable due to continuity of $S$. We have to show that $A \cap\left\{(\theta \cdot S)_{T}>-a\right\}$ has probability 0 . Define the entrance time $T^{\prime}=\inf \left\{t>0:(\theta \cdot S)_{t}=-a\right\} \wedge T$. Observe that the strategy $\pi=1_{A} 1_{\left.] T^{\prime}, T\right]} \theta$ satisfies

(i) $(\pi \cdot S)_{T}=1_{A}\left[(\theta \cdot S)_{T}-(\theta \cdot S)_{T^{\prime}}\right] \geqslant-a+a=0$,

(ii) $P\left((\pi \cdot S)_{T}>0\right)=P\left(T^{\prime}<T,(\theta \cdot S)_{T}>-a\right)=P\left(A \cap\left\{(\theta \cdot S)_{T}>-a\right\}\right)$.

If $P\left(A \cap\left\{(\theta \cdot S)_{T}>-a\right\}\right)>0$, then (i) and (ii) would qualify $\pi$ as an arbitrage opportunity. But this violates (NFLVR).

In a similar way we obtain

Proposition 1.5. Let $S$ be a continuous semimartingale satisfying (NFLVR). If $(\theta \cdot S)_{T} \geqslant-a$ a.s., then the process $\theta$ is a-admissible.

Proof. For every $\varepsilon>0$ define a stopping time by

$$
T_{\varepsilon}=\inf \left\{t>0:(\theta \cdot S)_{t}=-a-\varepsilon\right\} \wedge T .
$$

Suppose $\theta$ is not $a$-admissible. Then for some $\varepsilon>0$ we must have $P\left(T_{\varepsilon}<T\right)>0$. The strategy $\left.\pi=1_{]} T_{\varepsilon}, T\right] \theta$ satisfies

$$
\begin{aligned}
& (\pi \cdot S)_{T}=1_{\left\{T_{\varepsilon}<T\right\}}\left[(\theta \cdot S)_{T}-(\theta \cdot S)_{T_{\varepsilon}}\right] \geqslant 0, \\
& P\left((\pi \cdot S)_{T}>0\right)=P\left(T_{\varepsilon}<T\right)>0 .
\end{aligned}
$$

Hence $\pi$ is an arbitrage opportunity. But this is a contradiction to (NFLVR).

In the following proposition we approximate admissible general strategies by simple ones. 
Proposition 1.6. Let $S$ be a continuous semimartingale satisfying (NFLVR). For every a-admissible integrand $\theta$ there is a sequence of a-admissible simple processes $\left(\theta^{n}\right)_{n \geqslant 0}$ for which

$$
\left(\theta^{n} \cdot S\right)_{T} \longrightarrow(\theta \cdot S)_{T} \quad \text { a.s. }
$$

Proof. Let $\left(\pi^{n}\right)_{n \in \mathbb{N}}$ be an arbitrary sequence of simple integrands such that a.s. the trajectories of $\pi^{n} \cdot S$ converge uniformly to those of $\theta \cdot S$. For $n \in \mathbb{N}$, we put

$$
T_{n}=\inf \left\{t>0:\left(\pi^{n} \cdot S\right)_{t} \leqslant-a\right\} \wedge T .
$$

We first show that $T_{n}$ converges to $T$ a.s. on the set $\left\{(\theta \cdot S)_{T}>-a\right\}$.

According to Lemma 1.4 almost all $\omega \in\left\{(\theta \cdot S)_{T}>-a\right\}$ satisfy:

$$
(\theta \cdot S)_{t}>-a \text { for all } 0 \leqslant t \leqslant T .
$$

Since $\theta \cdot S$ is continuous, for almost all $\omega \in\left\{(\theta \cdot S)_{T}>-a\right\}$ there exists a $\delta=\delta(\omega)>0$ such that

$$
(\theta \cdot S)_{t}(\omega)>-a+\delta .
$$

Since $\left(\pi^{n} \cdot S\right)$ converges uniformly to $(\theta \cdot S)$, we find for almost every $\omega \in\left\{(\theta \cdot S)_{T}>-a\right\}$ some $n_{0}$ such that

$$
\left(\pi^{n} \cdot S\right)_{t}>-a \text { for all } 0 \leqslant t \leqslant T \text { and } n \geqslant n_{0} .
$$

It follows that $T_{n} \rightarrow T$ a.s. on the set $\left\{(\theta \cdot S)_{T}>-a\right\}$.

Furthermore, the simple processes $\theta^{n}=1_{\left[0, T_{n}\right]} \pi^{n}, n \in \mathbb{N}$, are obviously $a$-admissible and satisfy

$$
\begin{aligned}
\left|\left(\theta^{n} \cdot S\right)_{T}-(\theta \cdot S)_{T}\right| & =\left|\left(\theta^{n} \cdot S\right)_{T}-(\theta \cdot S)_{T}\right| 1_{\left\{T_{n}<T,(\theta \cdot S)_{T}>-a\right\}}+\left|\left(\theta^{n} \cdot S\right)_{T}-(\theta \cdot S)_{T}\right| 1_{\left\{T_{n}=T\right\}} \\
& \leqslant\left|\left(\theta^{n} \cdot S\right)_{T}-(\theta \cdot S)_{T}\right| 1_{\left\{T_{n}<T,(\theta \cdot S)_{T}>-a\right\}}+\left|\left(\pi^{n} \cdot S\right)_{T}-(\theta \cdot S)_{T}\right| .
\end{aligned}
$$

The first summand converges to 0 a.s., because $T_{n}$ converges to $T$ on the set $\left\{(\theta \cdot S)_{T}>-a\right\}$. Since the second summand also converges to 0 , we obtain that $\left(\theta^{n} \cdot S\right)_{T}$ converges to $(\theta \cdot S)_{T}$ a.s.

The preceding proposition now allows to prove the result we aim at if for a fixed $a$ we concentrate on $a$ admissible strategies.

Proposition 1.7. Let $S$ be a continuous semimartingale satisfying (NFLVR). If $a>0$ is such that $U(x-a)>-\infty$, then

$$
u_{a}(x)=\sup _{\mathcal{S} \ni \theta a \text {-adm. }} E\left[U\left(x+(\theta \cdot S)_{T}\right)\right]
$$

Proof. We have to prove that the right-hand side is not smaller than the left-hand side. Let therefore $\theta$ be an $a$ admissible integrand. Proposition 1.6 states that we can find a sequence of $a$-admissible simple processes $\left(\theta^{n}\right)_{n \in \mathbb{N}}$ such that $\left(\theta^{n} \cdot S\right)_{T} \rightarrow(\theta \cdot S)_{T}$ a.s. Since the random variables $U\left(\theta^{n} \cdot S\right)_{T}, n \in \mathbb{N}$, are bounded from below by $U(x-a)>-\infty$, we conclude by using Fatou's Lemma and the fact that $U$ is continuous on $\{y: U(y)>-\infty\}$ :

$$
\begin{aligned}
E\left[U\left(x+(\theta \cdot S)_{T}\right)\right] & =E\left[\lim _{n \rightarrow \infty} U\left(x+\left(\theta^{n} \cdot S\right)_{T}\right)\right] \leqslant \liminf _{n \rightarrow \infty} E\left[U\left(x+\left(\theta^{n} \cdot S\right)_{T}\right)\right] \\
& \leqslant \sup _{\mathcal{S} \ni \theta^{\prime} a \text {-adm. }} E\left[U\left(x+\left(\theta^{\prime} \cdot S\right)_{T}\right)\right]=u_{a}(x) .
\end{aligned}
$$

Remark. The proposition remains valid if $u_{a}(x)=\infty$.

We are now ready to state and prove the main result of this section. 
Theorem 1.8. Let $S$ be a continuous semimartingale satisfying (NFLVR). If $x>\sup \{y: U(y)=-\infty\}$, then

$$
u(x)=\sup _{\mathcal{S} \ni \theta \text { adm. }} E\left[U\left(x+(\theta \cdot S)_{T}\right)\right] .
$$

In particular, the maximal expected utility $u(x)$ is infinite if and only if $\sup _{\theta \ni \mathcal{S} \text { adm. }} E\left[U\left(x+(\theta \cdot S)_{T}\right)\right]=\infty$.

Proof. The proof will be executed in several steps. The utility functions admitted by the hypotheses above will be subdivided into several classes. This leads to distinguishing the following cases.

We start with

Case 1: $\{y: U(y)>-\infty\}=\mathbb{R}$.

Observe that the exponential utility function $U(x)=-\mathrm{e}^{-\alpha x}, x \in \mathbb{R}$, with $\alpha>0$, is covered by case 1 .

If the domain of $U$ is $\mathbb{R}$, any admissible strategy leads to a utility bounded from below. This is the main observation needed to prove the assertion in this case. Let $\zeta$ be any admissible integrand. According to Proposition 1.7 the expected utility $E U\left(x+(\zeta \cdot S)_{T}\right)$ is not greater than $\sup _{\mathcal{S} \ni \theta \text { adm. }} E\left[U\left(x+(\theta \cdot S)_{T}\right)\right]$. Hence we have

$$
u(x) \leqslant \sup _{\mathcal{S} \ni \theta \text { adm. }} E\left[U\left(x+(\theta \cdot S)_{T}\right)\right] .
$$

Since the left-hand side is obviously not smaller than the right-hand side, equality holds.

Case 2: $c=\sup \{y: U(y)=-\infty\} \in \mathbb{R}$ and $U(c)>-\infty$.

Think of the power utility function $U(x)=x^{\alpha} / \alpha, x \geqslant 0$, extended to be $-\infty$ for $x<0$, where $\alpha \in(0,1)$, as a typical example. If $\zeta$ is $(x-c)$-admissible, then by Proposition 1.7 the expected utility $E U\left(x+(\zeta \cdot S)_{T}\right)$ is dominated by $\sup _{\mathcal{S} \ni \theta \text { adm. }} E\left[U\left(x+(\theta \cdot S)_{T}\right)\right]$. Suppose now that $\zeta$ is not $(x-c)$-admissible. By Proposition 1.5 we have $(\theta \cdot S)_{T}<-x+c$ on a set of positive probability. Since $U(z)=-\infty$ for all $z<c$, the expected utility $E U\left(x+(\zeta \cdot S)_{T}\right)$ must equal $-\infty$. This provides the asserted equation in this case.

Case 3: $c=\sup \{y: U(y)=-\infty\} \in \mathbb{R}$ and $U(c)=-\infty$.

For example the logarithmic utility function is covered by this case.

To simplify notation we assume that $c=0$. We make use of Theorem 2.1 in [23], according to which the following statement holds true. If $u\left(x_{0}\right)<\infty$ for some $x_{0}>0$, then $u(x)<\infty$ for all $x>0$ and the function $u$ is continuously differentiable on $(0, \infty)$. With the help of this result we are able to prove the assertion in the given case.

Let $x>0$. Assume first that $u(x)<\infty$. Due to the quoted result $u$ is continuous on $(0, \infty)$. Hence for any $\varepsilon>0$ there exists a $0<y<x$ such that $u(x)-u(y)<\frac{\varepsilon}{2}$. Let $\zeta$ be an admissible strategy satisfying

$$
u(y)-E U\left(y+(\zeta \cdot S)_{T}\right) \leqslant \frac{\varepsilon}{2} .
$$

Proposition 1.5 guarantees that $\zeta$ is $y$-admissible. Starting with the initial wealth $x$, the utility process $U(x+$ $\left.(\zeta \cdot S)_{t}\right)$ will be bounded from below by the constant $D=U(x-y)>-\infty$. Again with Proposition 1.7 we obtain

$$
\begin{aligned}
u(x)-\sup _{\mathcal{S} \ni \theta \text { adm. }} E\left[U\left(x+(\theta \cdot S)_{T}\right)\right] & \leqslant u(x)-E U\left(x+(\zeta \cdot S)_{T}\right) \\
& =[u(x)-u(y)]+\left[u(y)-E U\left(x+(\zeta \cdot S)_{T}\right)\right] \\
& \leqslant[u(x)-u(y)]+\left[u(y)-E U\left(y+(\zeta \cdot S)_{T}\right)\right] \\
& \leqslant \frac{\varepsilon}{2}+\frac{\varepsilon}{2}=\varepsilon .
\end{aligned}
$$

Since $\varepsilon$ was arbitrary, the assertion follows.

Next suppose that $u(x)=\infty$. Then by the quoted Theorem 2.1 in [23] for $0<y<x$ the maximal utility $u(y)$ is also infinite. Choose $y$-admissible integrands $\theta^{n}, n \in \mathbb{N}$, such that

$$
E U\left(y+\left(\theta^{n} \cdot S\right)_{T}\right) \geqslant n \quad \text { for } n \in \mathbb{N} .
$$


Given the initial wealth $x$ we have

$$
U\left(x+\left(\theta^{n} \cdot S\right)_{t}\right) \geqslant U(x-y)>-\infty
$$

for all $t \geqslant 0, n \in \mathbb{N}$. Hence we can apply Proposition 1.7 to obtain for $n \in \mathbb{N}$

$$
\sup _{\mathcal{S} \ni \theta \text { adm. }} E\left[U\left(x+(\theta \cdot S)_{T}\right)\right] \geqslant E U\left(x+\left(\theta^{n} \cdot S\right)_{T}\right) \geqslant E U\left(y+\left(\theta^{n} \cdot S\right)_{T}\right) \geqslant n .
$$

This shows that $\sup _{\mathcal{S} \ni \theta \text { adm. }} E\left[U\left(x+(\theta \cdot S)_{T}\right)\right]=\infty$.

This completes the proof in the final case.

Combining Theorem 1.8 with the results of Subsection 1.1 we obtain a simple proof of the following generalization of one of the structure results for $S$ derived in the framework of Wiener space with tools of anticipative calculus in [8] that was alluded to in the introductory remarks.

Corollary 1.9. Let $S$ be an arbitrary adapted continuous process indexed by $[0, T], U$ a utility function with $\lim _{x \rightarrow \infty} U(x)=\infty$ and $x>\sup \{y: U(y)=-\infty\}$. If $\sup _{\mathcal{S} \ni \theta \text { adm. }} E\left[U\left(x+(\theta \cdot S)_{T}\right)\right]<\infty$, then $S$ is a semimartingale and the expected utility maximized over general admissible integrands is either infinite or given by $\sup _{\mathcal{S} \ni \theta \text { adm. }} E\left[U\left(x+(\theta \cdot S)_{T}\right)\right]$.

Proof. Suppose $S$ is a semimartingale. By applying similar arguments one can show that the conclusions of Lemma 1.1 and Proposition 1.2 hold for the set of general strategies, too. Hence, if $u(x)$ is finite, then $S$ satisfies (NFLVR) for general integrands. The result now follows by combining Corollary 1.3 and Theorem 1.8.

In Example 2.11 we shall exhibit a continuous price process $S$ for which $u(x)=\infty$, but $\sup _{\mathcal{S} \ni \theta \text { adm. }} E[U(x+$ $\left.(\theta \cdot S)_{T}\right)$ ] is finite. We close this section with an example inspired by Example 7.5 in [11] and showing that in Theorem 1.8 the requirement that $S$ is continuous cannot be dropped.

Example 1.10. Let $\left(X_{n}\right)_{n \in \mathbb{N}}$ be a sequence of Gaussian unit variables and $\left(\phi_{n}\right)_{n \in \mathbb{N}}$ a sequence of random variables satisfying $P\left(\phi_{n}=1\right)=2^{-n}$ and $P\left(\phi_{n}=0\right)=1-2^{-n}$. Furthermore suppose that $Z$ is a random variable with distribution $P(Z=a)=P(Z=b)=\frac{1}{2}$, where $0<a<1$ and $b>1$. We assume that all these random variables are independent. Choose an enumeration $\left(q_{n}\right)_{n \in \mathbb{N}}$ of the rationals in $[0,1[$. The process defined by

$$
S=1_{[0,1[}(t)+Z 1_{\{1\}}(t)+\sum_{\left\{n: q_{n} \leqslant t\right\}} \phi_{n} X_{n}, \quad 0 \leqslant t \leqslant 1,
$$

is cadlag. We start by showing that $S$ is a semimartingale satisfying the (NFLVR) property. For this purpose denote by $\widetilde{P}$ the restriction of $P$ to $\sigma(Z)$. It is obvious, that there is a probability measure $\widetilde{Q} \sim \widetilde{P}$ on $\sigma(Z)$ such that the expectation of $Z$ with respect to $\widetilde{Q}$ is equal to 1 . Note that the extension $\mathrm{d} Q=\frac{\mathrm{d} \widetilde{Q}}{\mathrm{~d} \widetilde{P}} \mathrm{~d} P$ is a probability measure such that

(i) $Q=\widetilde{Q}$ on $\sigma(Z)$,

(ii) $Q=P$ on $\sigma\left(\phi_{n} X_{n}, n \in \mathbb{N}\right)$ and

(iii) $Q \sim P$.

Hence the process $S$ is a $Q$-martingale with respect to its natural filtration. By the fundamental theorem of asset pricing (see Corollary 1.2 in [12]) this implies that $S$ is a semimartingale satisfying the (NFLVR) property.

As in example 7.5 in [11] one can show that $\theta=0$ is the only simple integrand which is admissible for $S$. Hence we have

$$
\sup _{\mathcal{S} \ni \theta \text { adm. }} E\left[U\left(x+(\theta \cdot S)_{1}\right)\right]=U(x) .
$$


However, the non-simple strategy $\theta=1_{\{1\}}$ has as final payoff

$$
x+(\theta \cdot S)_{1}=x+\left(S_{1}-S_{1-}\right)=x+(Z-1) \text { a.s. }
$$

If $\lim _{x \rightarrow \infty} U(x)=\infty$, choose $x, a$ and $b$ such that

$$
E U\left(x+(\theta \cdot S)_{1}\right)=\frac{1}{2} U(x+a-1)+\frac{1}{2} U(x+b-1)>U(x) .
$$

For example if $U=\log , x=1, a$ and $b$ are such that $a b=\mathrm{e}^{2}$, then

$$
E U\left(x+(\theta \cdot S)_{1}\right)=\frac{1}{2} \log (a)+\frac{1}{2} \log (b)=\frac{1}{2} \log (a b)=1>0=U(x) .
$$

Thus we have

$$
u(x) \neq \sup _{\mathcal{S} \ni \theta \text { adm. }} E\left[U\left(x+(\theta \cdot S)_{1}\right)\right] .
$$

\section{The expected logarithmic utility increment of an insider}

In this section we uniquely consider the case of logarithmic utility. So let

$$
U(x)= \begin{cases}\log x & \text { if } x>0 \\ -\infty & \text { if } x \leqslant 0\end{cases}
$$

throughout the section. According to the previous section bounded utility $u(x)<\infty$ for an agent with an information horizon $\mathcal{F}$ implies (NFLVR). Under this condition, Delbaen and Schachermayer [12] show that for continuous semimartingales $S$ the bounded variation part in the Doob-Meyer decomposition must be controlled by the martingale (uncertainty) part $M$ of $S$, i.e. there is an $\mathcal{F}$-predictable process $\alpha$ such that

$$
S=M+\alpha \cdot\langle M, M\rangle .
$$

Equipped with this knowledge we now return to the setting of a financial market with small agents, i.e. agents not able to influence the price dynamics, possessing asymmetric information, to perform the second part of our task of calculating the expected utility increment of a better informed agent in a fairly general setting using basic and natural tools, in particular generalizing Theorem 3.7 of [8]. So we assume that each of the agents (regular and better informed trader) takes his portfolio decisions on the basis of his individual information horizon, given by different filtrations $\mathcal{F}$ and $\mathcal{G}$. We just suppose that the insider's filtration is bigger, but do not specify at all what the sources for the additional information in $\mathcal{G}$ are. The asset price process $S$ will be assumed to be continuous. The starting point of our analysis according to the previous section have to be the agents who possess finite utility from investing into $S$ on the basis of their knowledge, which is therefore described by the following type of filtrations.

Definition 2.1. Let $\mathcal{H}$ be a filtration satisfying the usual conditions, $S$ a $\mathcal{H}$-semimartingale with decomposition $S=M+\alpha \cdot\langle M, M\rangle$, and $L_{\mathcal{H}}^{2}\left(P_{M}\right)$ the space of all $\mathcal{H}$-predictable processes $\gamma$ such that $E \int_{0}^{T} \gamma^{2} \mathrm{~d}\langle M, M\rangle<\infty$. The filtration $\mathcal{H}$ will be called finite utility filtration for $S$ if $\alpha$ belongs to $L_{\mathcal{H}}^{2}\left(P_{M}\right)$.

Of course, a finite utility filtration for fixed $S$ should just be a filtration for which the expected logarithmic utility of an agent who makes his portfolio decisions depend on this information flow is finite. We shall see below in the second subsection that this intuitive notion is consistent with the above definition.

Note that a finite utility filtration for $S$ may not be a finite utility filtration for a different process. Nevertheless we will often omit the process in the definition since we are always referring to a fixed $S$.

Now let $\mathcal{F}$ be a finite utility filtration for $S$. Assume that a better informed agent's filtration is given by $\mathcal{G}$. We shall see in the third subsection below that the logarithmic utility increment of this agent with respect to the 
non-informed one only depends on a quantity which we shall call information drift according to the following definition.

Definition 2.2. Let $\mathcal{F}$ be a finite utility filtration. Suppose that $\mathcal{G}$ is a filtration such that $\mathcal{F}_{t} \subset \mathcal{G}_{t}$ for all $t \in[0, T]$ and $\mu$ is a $\mathcal{G}$-predictable process satisfying

$$
M-\int_{0} \mu_{t} \mathrm{~d}\langle M, M\rangle_{t} \quad \text { is a } \mathcal{G} \text {-local martingale. }
$$

Then $\mu$ is called information drift (see [18]) of $\mathcal{G}$ with respect to $\mathcal{F}$.

To get a general description of this fundamental quantity, let us consider the situation in which both agents, the uninformed and the insider, are acting on finite utility filtrations. So let $\mathcal{F}$ and $\mathcal{G}$ be two finite utility filtrations for $S$. We denote by

$$
S=M+\alpha \cdot\langle M, M\rangle
$$

the semimartingale decomposition with respect to $\mathcal{F}$ and by

$$
S=N+\beta \cdot\langle N, N\rangle
$$

the decomposition with respect to $\mathcal{G}$. Obviously,

$$
\langle M, M\rangle=\langle S, S\rangle=\langle N, N\rangle
$$

and therefore Eqs. (5) and (6) imply

$$
M=N-(\alpha-\beta) \cdot\langle M, M\rangle \quad \text { a.s. }
$$

If $\mathcal{F}_{t} \subset \mathcal{G}_{t}$ for all $t \geqslant 0$, Eq. (7) can be interpreted as the semimartingale decomposition of $M$ with respect to $\mathcal{G}$, and $\mu=\alpha-\beta$ as the corresponding information drift.

Our main result will show that in this general setting the finite utility advantage of an insider compared to the regular trader is given by

$$
\frac{1}{2} E \int_{0}^{T} \mu_{s}^{2} \mathrm{~d}\langle M, M\rangle_{s},
$$

if $\mu$ is the information drift obtained by passing from $\mathcal{F}$ to $\mathcal{G}$.

To prove this formula, we shall proceed in three steps. The first one is of more auxiliary character.

\subsection{Infinite utility and drift}

We shall start by establishing a relationship between the intensity of the intrinsic $\operatorname{drift} \alpha \cdot\langle M, M\rangle$ of $S$ and the boundedness of expected utility. We shall prove that if this drift collects infinite mass on $[0, T]$ with positive probability, then expected logarithmic utility will be infinite. This will be helpful in the direct computation of expected logarithmic utility in the following subsection. Due to close connections between (NFLVR) and finite utility, explained in Section 1, our treatment will in some parts heavily rely on similar arguments in Delbaen and Schachermayer [12].

In the following lemma a link between the infinite intrinsic drift and the existence of admissible strategies inducing large wealths is established.

Lemma 2.3. Suppose $P\left(\int_{0}^{T} \alpha^{2} \mathrm{~d}\langle M, M\rangle=\infty\right)=\eta>0$. Then for all a, $\xi>0$ we can find an a-admissible integrand $\theta$ such that $P\left((\theta \cdot S)_{T} \geqslant 1\right) \geqslant \eta-\xi$. 
Proof. The proof is essentially the same as the one of Lemma 3.8 in [12], and is therefore omitted.

As an immediate consequence of the preceding, infinite drift with positive probability entails that free lunches are possible.

Corollary 2.4. If $\int_{0}^{T} \alpha^{2} \mathrm{~d}\langle M, M\rangle=\infty$ on a set with positive probability, then $S$ satisfies (FLVR).

For later use we are mainly interested in another consequence of the lemma. It says that infinite drift with positive probability also implies that the expected utility becomes infinite.

Theorem 2.5. If $U=\log$ and $\int_{0}^{T} \alpha^{2} \mathrm{~d}\langle M, M\rangle=\infty$ on a set with positive probability, then for all $a>0$ and $x>0$ we have

$$
u_{a}(x)=\infty
$$

Proof. By eventually reducing $a$ we may assume that $0<a<x$. By Lemma 2.3 there is an $\alpha>0$ and a sequence $\left(\theta^{n}\right)_{n \in \mathbb{N}}$ of $a$-admissible integrands satisfying

$$
P\left(\left(\theta^{n} \cdot S\right)_{T} \geqslant n\right)>\alpha .
$$

Since $\lim _{x \rightarrow \infty} \log (x)=\infty$, we obtain

$$
\liminf _{n \rightarrow \infty} E U\left(x+\left(\theta^{n} \cdot S\right)_{T}\right) \geqslant \liminf _{n \rightarrow \infty} \log (x+n) \alpha+\log (x-a)(1-\alpha)=\infty,
$$

which proves the theorem.

Remark. The theorem neither follows from the preceding corollary nor from the 'Immediate Arbitrage Theorem' of Delbaen and Schachermayer in [12]. This is because there are situations where (NA) is violated, but $u_{a}(x)$ is finite for some $a$ (see examples below).

\subsection{Logarithmic utility of an agent}

Our second step consists in computing explicitly the expected logarithmic utility of an agent acting on the basis of some filtration $\mathcal{F}$, with respect to which $S$ possesses a Doob-Meyer decomposition (4). We shall prove implicitly that it only depends on the drift density $\alpha$, and is given by a formula which in case there is a martingale measure for $S$ is provided by the general analysis of Kramkov and Schachermayer [23]. We shall give a derivation of the formula which is valid irrespective of whether (NFLVR) holds, provided only that (4) is guaranteed. For example, if $\mathcal{F}$ is the Wiener filtration initially enlarged by the maximum of the Wiener process on [0,T], (NFLVR) is violated, whereas $S$ is a semimartingale satisfying (4) with a well known $\alpha$ (see [19]). The method we employ consists in using the linear stochastic equation link allowing to describe the optimal portfolio $\theta^{*}$ as a function of the drift process $\alpha$ in a general framework. In Subsubsection 2.2.1, we shall consider the case of positive wealth, while in Subsubsection 2.2.2 we extend the results to the case in which wealth may become negative.

\subsubsection{Maximal utility if wealth stays positive}

If wealth always stays positive, we may consider the following class of admissible strategies.

Definition 2.6. Let $a>0$ be given. An $S$-integrable and predictable process $\theta$ is called $a$-superadmissible if almost surely $(\theta \cdot S)_{t}>-a$ for all $t \in[0, T]$. 
Our goal is to find

$$
u^{+}(x)=\sup _{\mathcal{A} \ni \theta} E \log \left(x+(\theta \cdot S)_{T}\right) .
$$

It will be helpful to express the right-hand side in terms of the so-called optimal portfolio, i.e. the process $\theta^{*} \in \mathcal{A}$ which satisfies $u^{+}(x)=E \log \left(x+\left(\theta^{*} \cdot S\right)_{T}\right)$. Before we can show that the optimal portfolio exists and may be expressed as a function of $\alpha$, we have to prove some auxiliary results which will ultimately turn out to present the optimal portfolio as the unique solution of a linear stochastic equation. Recall that we do not assume (NFLVR) here. We start by proving

Proposition 2.7. Suppose $\int_{0}^{T} \alpha_{s}^{2} \mathrm{~d}\langle M, M\rangle_{s}<\infty$ a.s. If $\pi$ is a predictable and S-integrable process, then the product $\mathcal{E}(\pi \cdot S) \mathcal{E}(-\alpha \cdot M)$ is a local martingale.

Proof. We use Yor's addition formula

$$
\mathcal{E}(X) \mathcal{E}(Y)=\mathcal{E}(X+Y+\langle X, Y\rangle)
$$

for two continuous semimartingales (see e.g. [15], p. 374). It implies

$$
\mathcal{E}(\pi \cdot S) \mathcal{E}(-\alpha \cdot M)=\mathcal{E}((\pi-\alpha) \cdot M),
$$

hence, the result.

Remark. Proposition 2.7 states that $\mathcal{E}(-\alpha \cdot M)$ is a strict martingale density for $\mathcal{E}(\pi \cdot S)$ in the sense of Schweizer [30].

Lemma 2.8. Suppose that $x>0$ and $E \int_{0}^{T} \alpha_{s}^{2} \mathrm{~d}\langle M, M\rangle_{s}<\infty$. The process $\theta^{*}=x \alpha \mathcal{E}(\alpha \cdot S)$ is $x$-superadmissible, belongs to $\mathcal{A}$ and solves the integral equation

$$
\theta_{t}^{*}=\alpha_{t}\left(x+\int_{0}^{t} \theta_{r}^{*} \mathrm{~d} S_{r}\right), \quad 0 \leqslant t \leqslant T
$$

Proof. We observe that the process $\theta^{*}=x \alpha \mathcal{E}(\alpha \cdot S)$ is predictable and satisfies for all $t \in[0, T]$

$$
x+\left(\theta^{*} \cdot S\right)_{t}=x+x \int_{0}^{t} \alpha_{r} \mathcal{E}(\alpha \cdot S)_{r} \mathrm{~d} S_{r}=x\left(1+\int_{0}^{t} \alpha_{r} \mathcal{E}(\alpha \cdot S)_{r} \mathrm{~d} S_{r}\right)=x \mathcal{E}(\alpha \cdot S)_{t}>0
$$

This yields that $\theta^{*}$ is $x$-superadmissible. At the same time, multiplying both extreme terms by $\alpha_{t}$ shows that $\theta^{*}$ solves (8).

The expression

$$
E \log \left(x+\left(\theta^{*} \cdot S\right)_{T}\right)=\log x+E(\alpha \cdot S)_{T}-\frac{1}{2} E \int_{0}^{T} \alpha_{s}^{2} \mathrm{~d}\langle M, M\rangle_{S}
$$

makes sense due to the integrability condition $E \int_{0}^{T} \alpha_{s}^{2} \mathrm{~d}\langle M, M\rangle_{s}<\infty$. Hence $\theta^{*}$ belongs to $\mathcal{A}$.

We now state the main result of this subsubsection. It generalizes Theorem 3.5 of [3], where it was proved in the special case of a semimartingale given by an SDE. 
Theorem 2.9. For any $x>0$ the following equation holds

$$
u^{+}(x)=\log x+\frac{1}{2} E \int_{0}^{T} \alpha_{s}^{2} \mathrm{~d}\langle M, M\rangle_{s} .
$$

If $E \int_{0}^{T} \alpha_{s}^{2} \mathrm{~d}\langle M, M\rangle_{s}<\infty$, then the process $\theta^{*}=x \alpha \mathcal{E}(\alpha \cdot S)$ is the unique optimal portfolio.

Proof. We first assume that $E \int_{0}^{T} \alpha_{s}^{2} \mathrm{~d}\langle M, M\rangle_{s}<\infty$.

Let $\theta \in \mathcal{A}$ be $x$-superadmissible. Then $x+(\theta \cdot S)_{t}>0$ a.s. for all $t \in[0, T]$ and hence we can define a new process by

$$
\pi_{t}=\frac{\theta_{t}}{x+(\theta \cdot S)_{t}}, \quad 0 \leqslant t \leqslant T
$$

Since $\pi$ is predictable, the integral $\pi \cdot S$ is defined.

The SDE

$$
\begin{aligned}
& Y_{0}=x, \\
& \mathrm{~d} Y_{t}=\pi_{t} Y_{t} \mathrm{~d} S_{t}=Y_{t} \mathrm{~d}(\pi \cdot S)_{t}
\end{aligned}
$$

is uniquely solved by the process $Y=x \mathcal{E}(\pi \cdot S)$. On the other hand the process $x+(\theta \cdot S)_{t}$ is also easily seen to be a solution. By uniqueness this implies

$$
x+(\theta \cdot S)=x \mathcal{E}(\pi \cdot S) .
$$

In the next step we will show that the expected logarithmic utility of $x+(\theta \cdot S)_{T}$ is not exceeded by $\log x+$ $\frac{1}{2} E \int_{0}^{T} \alpha_{s}^{2} \mathrm{~d}\langle M, M\rangle_{s}$. Applying the inequality $\log z \leqslant z-1$, valid for positive $z$, to the product of two positive numbers $a, b$ we get the inequality

$$
\log a \leqslant a b-\log b-1 .
$$

If we take $a=x \mathcal{E}(\pi \cdot S)$ and $b=\frac{1}{x} \mathcal{E}(-\alpha \cdot M)$ we obtain

$$
\log x \mathcal{E}(\pi \cdot S) \leqslant \mathcal{E}(\pi \cdot S) \mathcal{E}(-\alpha \cdot M)-\log \frac{1}{x} \mathcal{E}(-\alpha \cdot M)-1 .
$$

By Proposition 2.7 the product $\mathcal{E}(\pi \cdot S) \mathcal{E}(-\alpha \cdot M)$ is a local martingale. Since it is nonnegative, it is also a supermartingale and therefore by (10)

$$
\begin{aligned}
E\left[\log \left(x+(\theta \cdot S)_{T}\right)\right] & =E\left[\log x \mathcal{E}(\pi \cdot S)_{T}\right] \leqslant E\left[\mathcal{E}(\pi \cdot S)_{T} \mathcal{E}(-\alpha \cdot M)_{T}-\log \frac{1}{x} \mathcal{E}(-\alpha \cdot M)_{T}-1\right] \\
& \leqslant-E\left[\log \frac{1}{x} \mathcal{E}(-\alpha \cdot M)_{T}\right]=\log x-E\left[-\int_{0}^{T} \alpha_{t} \mathrm{~d} M_{t}-\frac{1}{2} \int_{0}^{T} \alpha^{2} \mathrm{~d}\langle M, M\rangle\right] \\
& =\log x+\frac{1}{2} E \int_{0}^{T} \alpha^{2} \mathrm{~d}\langle M, M\rangle
\end{aligned}
$$

This implies

$$
u^{+}(x) \leqslant \log x+\frac{1}{2} E \int_{0}^{T} \alpha^{2} \mathrm{~d}\langle M, M\rangle .
$$

Before we prove that in fact equality holds, we note 


$$
E \log \left(x \mathcal{E}(\alpha \cdot S)_{T}\right)=\log x+\frac{1}{2} E \int_{0}^{T} \alpha^{2} \mathrm{~d}\langle M, M\rangle .
$$

Therefore it is enough to show that there is a process $\theta$ such that $E \log \left(x+(\theta \cdot S)_{T}\right)=E \log \left(x \mathcal{E}(\alpha \cdot S)_{T}\right)$.

According to Lemma 2.8 the process $\theta^{*}=x \alpha \mathcal{E}(\alpha \cdot S)$ belongs to $\mathcal{A}$, is $x$-superadmissible and satisfies

$$
\alpha=\frac{\theta^{*}}{x+\left(\theta^{*} \cdot S\right)},
$$

from which we deduce

$$
x+\left(\theta^{*} \cdot S\right)_{t}=x \mathcal{E}(\alpha \cdot S)_{t} .
$$

This proves the theorem in the case where $E \int_{0}^{T} \alpha_{s}^{2} \mathrm{~d}\langle M, M\rangle_{s}<\infty$.

We now claim that Eq. (9) is still true if $E \int_{0}^{T} \alpha_{s}^{2} \mathrm{~d}\langle M, M\rangle_{s}=\infty$. Suppose first $\int_{0}^{T} \alpha_{s}^{2} \mathrm{~d}\langle M, M\rangle_{s}=\infty$ on a set with positive probability. Then Theorem 2.5 yields $u^{+}(x)=\infty$.

On the other hand, if $\int_{0}^{T} \alpha_{s}^{2} \mathrm{~d}\langle M, M\rangle_{s}<\infty$ almost surely, we can find an increasing sequence of stopping times $\left(T_{n}\right)_{n \in \mathbb{N}}$ such that $T_{n} \rightarrow T$ and

$$
E \int_{0}^{T_{n}} \alpha_{s}^{2} \mathrm{~d}\langle M, M\rangle_{s}<\infty
$$

With the first part of the proof we deduce

$$
u^{+}(x) \geqslant \log x+\frac{1}{2} E \int_{0}^{T_{n}} \alpha_{s}^{2} \mathrm{~d}\langle M, M\rangle_{s}
$$

for all $n \in \mathbb{N}$. By Beppo-Levi the right-hand side goes to infinity as $n \rightarrow \infty$. Hence $u^{+}(x)=\infty$, which completes the proof.

\subsubsection{Maximal utility if wealth may become negative}

Here we allow the wealth process to take negative values and again deduce the desired formula for $u(x)$.

Let $S=M+\alpha \cdot\langle M, M\rangle$ be a continuous semimartingale satisfying (NFLVR). If $\theta \in \mathcal{A}$ is not $x$-superadmissible, then by Lemma 1.4

$$
(\theta \cdot S)_{T} \leqslant-x
$$

on a set of positive probability. But this implies $E \log \left(x+(\theta \cdot S)_{T}\right)=-\infty$ and therefore $u(x)=u^{+}(x)$. Hence we have shown

Theorem 2.10. Let $S$ be a continuous semimartingale satisfying (NFLVR). The maximal expected logarithmic utility is given by

$$
u(x)=\log x+\frac{1}{2} E \int_{0}^{T} \alpha_{s}^{2} \mathrm{~d}\langle M, M\rangle_{s} .
$$

Remark. Kramkov and Schachermayer [23] show that under the assumption of (NFLVR) a more general result can be obtained. They give explicit formulas for the maximal expected utility not only for the logarithm but for a large class of utility functions. We mention that $E \int_{0}^{T} \alpha_{s}^{2} \mathrm{~d}\langle M, M\rangle_{s}<\infty$ does not imply the (NFLVR) property. In the following examples the integral of the drift is finite, but arbitrage is possible and hence $u(x)$ is infinite (see Proposition 1.2). Hence the assumption of (NFLVR) in Theorem 2.10 cannot be dropped. 
Example 2.11. Let $S$ be a $B E S^{3}$ process starting in $x>0$. It is known that $S$ solves the equation

$$
S_{t}=x+B_{t}+\int_{0}^{t} S_{u}^{-1} \mathrm{~d} u, \quad 0 \leqslant t,
$$

where $\left(B_{t}\right)$ is a Brownian motion (see Proposition 3.3, Chapter VI in [28]). It is straightforward to show that

$$
E \int_{0}^{T} S_{u}^{-2} \mathrm{~d} u<\infty
$$

and hence, by Theorem 2.9, $u^{+}(x)$ is finite, too. On the other hand Delbaen and Schachermayer prove in [13] that $S$ allows arbitrage.

Moreover, this example shows that the assumption (NFLVR) cannot be dropped in Theorem 1.8: It is known that there are no simple arbitrage strategies (see [13]). Hence every simple strategy $\theta$ satisfying $U\left(x+(\theta \cdot S)_{T}\right)>0$, a.s., must be $x$-superadmissible (else one can construct a simple arbitrage strategy). Consequently

$$
\sup _{\mathcal{S} \ni \theta \text { adm. }} E\left[U\left(x+(\theta \cdot S)_{T}\right)\right] \leqslant u^{+}(x)<\infty .
$$

Since $S$ allows arbitrage for general integrands, we have $u(x)=\infty$. Thus Theorem 1.8 does not hold without the assumption (NFLVR).

Situations where the trader has finite utility $u^{+}(x)$, but (NFLVR) is not satisfied, can easily arise on markets with insiders. An insider acts using information from an enlarged filtration. As in the following example, this produces sources for possible arbitrage which, in contrast to the previous example, are very explicit.

Example 2.12. Let $W$ be a Brownian motion on some probability space $(\Omega, F, P)$. We denote by $\left(\mathcal{F}_{t}\right)_{t \geqslant 0}$ the completed filtration generated by $W$. We will study the price process

$$
S_{t}=\mathcal{E}(W)_{t}, \quad t \geqslant 0,
$$

not under $\left(\mathcal{F}_{t}\right)_{t \geqslant 0}$ but with respect to a larger filtration. Choose for example $T=1$, let $a, b \in \mathbb{R}$ such that $a<b$, let $G=1_{[a, b]}\left(W_{1}\right)$, and take the right continuous and completed version of $\mathcal{G}_{t}=\mathcal{F}_{t} \vee \sigma(G), t \in[0,1]$. It has been shown in [3] that an agent in this filtration possesses finite logarithmic utility, if wealth has to be positive. $u^{+}(x)$ is given by the entropy of $G$, or, alternatively, by $\frac{1}{2} E \int_{0}^{1} \alpha_{s}^{2} \mathrm{~d} s$ with the corresponding information drift $\alpha$.

We will see now that there are arbitrage strategies. Define a stopping time by

$$
T=\inf \left\{t \geqslant 0: W_{t} \leqslant a-1\right\} \wedge 1 .
$$

The strategy $\theta=1_{\left\{W_{1} \in[a, b]\right\}} 1_{] T, 1]}$ is admissible, because

$$
(\theta \cdot S)_{t} \geqslant-\mathrm{e}^{a-1}, \quad 0 \leqslant t \leqslant 1 .
$$

Furthermore $\theta$ satisfies

(i) $(\theta \cdot S)_{1}=1_{\left\{W_{1} \in[a, b]\right\}}\left(S_{1}-S_{T}\right) \geqslant 0$ and

(ii) $P\left((\theta \cdot S)_{1}>0\right)=P\left(T<1, W_{1} \in[a, b]\right)>0$,

which shows that $\theta$ is an arbitrage strategy. In particular $S$ does not have the (NFLVR) property.

Remark. Assume that an agent with information horizon $\mathcal{F}$ possesses bounded logarithmic utility, i.e. $u(x)<\infty$. According to Section 1 we therefore know that $S$ enjoys the (NFLVR) property, and thus it is a semimartingale 
with Doob-Meyer decomposition of the form (4) due to [12]. Now suppose that the maximal expected logarithmic utility is bounded only if the wealth of the agent has to stay positive. In this case the (NFLVR) condition does not necessarily hold. However, as will be shown in Ankirchner [4], a decomposition (4) still exists.

\subsection{Computation of the utility increment}

Now we return to the situation in which two agents take action with respect to two finite utility filtrations $\mathcal{F}$ and $\mathcal{G}$ for $S$. Recall the Doob-Meyer decompositions

$$
S=M+\alpha \cdot\langle M, M\rangle=N+\beta \cdot\langle M, M\rangle
$$

with an $\mathcal{F}$-martingale $M$, and a $\mathcal{G}$-martingale $N$, so that the information drift when passing from $\mathcal{F}$ to $\mathcal{G}$ is given by

$$
\mu=\alpha-\beta \text {. }
$$

Assume $\mathcal{H}$ is a given filtration. Given an initial wealth $x>0$, we denote by $u^{+}(\mathcal{H}, x)$ the corresponding maximal expected utility if wealth has always to be positive. Note that if $\mathcal{H}$ is a finite utility filtration, $u^{+}(\mathcal{H}, x)$ is finite for all $x>0$ (see Theorem 2.9).

A bigger filtration must clearly lead to a bigger maximal utility. The following main theorem will quantify this increase and describe the utility increment precisely as a function of the information drift $\mu$ of $\mathcal{G}$ with respect to $\mathcal{F}$.

Theorem 2.13. Let $\mathcal{F}$ and $\mathcal{G}$ be finite utility filtrations for $S$, with $\mathcal{F}_{t} \subset \mathcal{G}_{t}$ for all $t \in[0, T]$. Let $\mu$ denote the information drift of $\mathcal{G}$ with respect to $\mathcal{F}$. Then for any $x>0$

$$
u^{+}(\mathcal{G}, x)-u^{+}(\mathcal{F}, x)=\frac{1}{2} E \int_{0}^{T} \mu^{2} \mathrm{~d}\langle M, M\rangle
$$

Proof. Since $\alpha$ and $\beta$ are in $L^{2}\left(P_{M}\right)$, we can write

$$
\frac{1}{2} E \int_{0}^{T}(\beta-\alpha)^{2} \mathrm{~d}\langle M, M\rangle=\frac{1}{2} E \int_{0}^{T}\left(\beta^{2}-\alpha^{2}\right) \mathrm{d}\langle M, M\rangle+E \int_{0}^{T}\left(\alpha^{2}-\alpha \beta\right) \mathrm{d}\langle M, M\rangle .
$$

Since $\alpha$ is $\mathcal{F}$-, hence $\mathcal{G}$-adapted, it follows from (7)

$$
E \int_{0}^{T}\left(\alpha^{2}-\alpha \beta\right) \mathrm{d}\langle M, M\rangle=E \int_{0}^{T} \alpha \mathrm{d} N-E \int_{0}^{T} \alpha \mathrm{d} M=0 .
$$

Hence

$$
\frac{1}{2} E \int_{0}^{T}(\beta-\alpha)^{2} \mathrm{~d}\langle M, M\rangle=\frac{1}{2} E \int_{0}^{T}\left(\beta^{2}-\alpha^{2}\right) \mathrm{d}\langle M, M\rangle=u^{+}(\mathcal{G}, x)-u^{+}(\mathcal{F}, x) .
$$

Relative information drifts are additive with respect to successive refinements of filtrations. Indeed, let $\mathcal{F}, \mathcal{G}$ and $\mathcal{H}$ be three finite utility filtrations such that $\mathcal{F}_{t} \subset \mathcal{G}_{t} \subset \mathcal{H}_{t}$ for all $t \in[0, T]$. Suppose that $\mu$ is the information drift of $\mathcal{G}$ with respect to $\mathcal{F}$. Then by the definition we know that $\widetilde{M}=M-\mu \cdot\langle M, M\rangle$ is a $\mathcal{G}$-local martingale. If $\lambda$ is the information drift of $\mathcal{H}$ with respect to $\mathcal{G}$, then $\widetilde{M}-\lambda \cdot\langle M, M\rangle$ is a $\mathcal{H}$-local martingale. As a consequence $\kappa=\mu+\lambda$ is the information drift of $\mathcal{H}$ with respect to $\mathcal{F}$. We obtain 
Proposition 2.14. The information drift of $\mathcal{H}$ w.r.t. $\mathcal{F}$ is the sum of the information drift of $\mathcal{G}$ w.r.t. $\mathcal{F}$ and the information drift of $\mathcal{H}$ w.r.t. $\mathcal{G}$.

In the same situation, we will now show that the information drift of $\mathcal{G}$ with respect to $\mathcal{F}$ can be expressed as the predictable projection of the information drift of $\mathcal{H}$ with respect to $\mathcal{F}$ onto the space $L_{\mathcal{G}}^{2}\left(P_{M}\right)$. To this end, we need the following useful property.

Lemma 2.15. Let $\gamma \in L_{\mathcal{H}}^{2}\left(P_{M}\right)$ and let ${ }^{p} \gamma$ denote the projection of $\gamma$ onto $L_{\mathcal{G}}^{2}\left(P_{M}\right)$. Then

$$
E\left[\int_{t}^{\infty} \gamma_{s} \mathrm{~d}\langle M, M\rangle_{s} \mid \mathcal{G}_{t}\right]=E\left[\int_{t}^{\infty} p \gamma_{s} \mathrm{~d}\langle M, M\rangle_{s} \mid \mathcal{G}_{t}\right]
$$

for all $t \geqslant 0$.

Proof. Let $t \geqslant 0$ and $A \in \mathcal{G}_{t}$. Note that the process $1_{A} 1_{] t, \infty[}$ is $\mathcal{G}$-predictable. Since $\left(\gamma-{ }^{p} \gamma\right)$ is orthogonal to $L_{\mathcal{G}}^{2}\left(P_{M}\right)$, we have

$$
0=E \int_{0}^{\infty} 1_{A} 1_{] t, \infty[}(s)\left(\gamma_{s}-{ }^{p} \gamma_{s}\right) \mathrm{d}\langle M, M\rangle_{s}=E\left[1_{A} \int_{t}^{\infty}\left(\gamma_{s}-{ }^{p} \gamma_{s}\right) \mathrm{d}\langle M, M\rangle_{s}\right],
$$

and thus the result.

Equipped with these prerequisites we can state our theorem about the orthogonal projection property of information drifts. Recall that $\mathcal{F} \subset \mathcal{G} \subset \mathcal{H}$ are finite utility filtrations and $S=M+\alpha \cdot\langle M, M\rangle$ is the decomposition w.r.t. $\mathcal{F}$.

Theorem 2.16. Let $\kappa \in L_{\mathcal{H}}^{2}\left(P_{M}\right)$ be the information drift of $\mathcal{H}$ with respect to $\mathcal{F}$. Then the orthogonal projection of $\kappa$ onto $L_{\mathcal{G}}^{2}\left(P_{M}\right)$ is the information drift of $\mathcal{G}$ with respect to $\mathcal{F}$.

Proof. We may assume, by localizing the processes with some stopping time, that both $M$ and $\kappa \cdot\langle M, M\rangle$ are bounded. Let ${ }^{p} \kappa$ denote the orthogonal projection of $\kappa$ onto $L_{\mathcal{G}}^{2}\left(P_{M}\right)$. We have to show that $M-{ }^{p} \kappa \cdot\langle M, M\rangle$ is a $\mathcal{G}$-martingale. Choose $0 \leqslant s<t$ and a set $A \in \mathcal{G}_{s}$. Since $\mathcal{G}$ is a sub-filtration of $\mathcal{H}$ we obtain, using Lemma 2.15,

$$
E\left(1_{A}\left(M_{t}-M_{s}\right)\right)=E\left(1_{A} \int_{s}^{t} \kappa_{r} \mathrm{~d}\langle M, M\rangle_{r}\right)=E\left(1_{A} \int_{s}^{t} p_{\kappa_{r}} \mathrm{~d}\langle M, M\rangle_{r}\right) .
$$

This proves the claim.

In particular we have

Corollary 2.17. If $\beta$ is the information drift of some finite utility filtration $\mathcal{K}$ with respect to $\mathcal{F}$, then $\beta$ is orthogonal to $L_{\mathcal{F}}^{2}\left(P_{M}\right)$.

\section{Additional utility of an insider on a complete market}

The main aim of this section is to describe the additional utility of an insider with respect to a regular trader for fairly arbitrary utility functions. Besides, we shall briefly discuss always optimal strategies, i.e. strategies that 
optimize expected utility from terminal wealth if any time $t$ in the trading interval may be chosen as terminal. Again, the setting is very general: we specify the information advantage between the insider with filtration $\mathcal{G}$ and the regular trader with filtration $\mathcal{F}$ just by the relative information drift. As opposed to the previous section, we however assume the market to be complete here, so that we may invoke the general results by Kramkov and Schachermayer [23] about maximal utility. As usual, we assume the asset price process $S$ indexed by [0,T] to be continuous. Completeness entails that there is a unique equivalent local martingale measure, which we will denote by $Q$. By the fundamental theorem of asset pricing the NFLVR property holds and hence we may decompose $S$ into

$$
S=M+\alpha \cdot\langle M, M\rangle,
$$

where $M$ is a $P$-local martingale and $\alpha$ an $\mathcal{F}$-predictable process. The Radon-Nikodym density of the martingale measure given $P$ is known to be described by the exponential of $\alpha \cdot M$ :

$$
\left.\frac{\mathrm{d} Q}{\mathrm{~d} P}\right|_{\mathcal{F}_{t}}=\mathcal{E}(-\alpha \cdot M)_{t}, \quad t \in[0, T]
$$

(see [14]).

In the following we shall abbreviate

$$
Z=\mathcal{E}(-\alpha \cdot M) \text {. }
$$

Let us next describe the class of utility functions for which the maximal expected utility can be explicitly calculated by means of $Z$. Let $U$ be strictly increasing, strictly concave and continuously differentiable on $(0, \infty)$. Furthermore we assume that $U$ satisfies

$$
\lim _{x \rightarrow 0^{+}} U^{\prime}(x)=\infty \text { and } \lim _{x \rightarrow \infty} U^{\prime}(x)=0
$$

and that

$$
u\left(x_{0}\right)<\infty \text { for some } x_{0}>0 .
$$

On $(0, \infty)$ the derivative of $U$ has an inverse function, which we will denote by $I$. Observe that $I$ is a function with domain $(0, \infty)$ and with range $(0, \infty)$. The following formula for the maximal expected utility is obtained by Kramkov, Schachermayer [23].

Theorem 3.1 (Theorem 2.0 in [23]). Assume that the conditions (13) and (14) are satisfied. For all $x>0$ we have

$$
u(x)=E U\left(I\left(y Z_{T}\right)\right),
$$

where $y$ is the real number satisfying $E\left[Z_{T} I\left(y Z_{T}\right)\right]=x$. Furthermore the process $I\left(y Z_{t}\right)$ is a uniformly integrable martingale under $Q$, hence $x=I(y)$, and consequently $y$ does not depend on the time horizon $T$.

\subsection{Always optimal strategies}

The maximal expected utility $u(x)$ depends of course on the time interval in which the traders are allowed to act. We will denote by $u_{t}(x)$ the maximal expected utility of a trader of initial wealth $x$ who is not allowed to hold any shares of the stock after time $t \leqslant T$, i.e.

$$
u_{t}(x)=\sup _{\theta \in \mathcal{A}} E U\left(x+\left(\theta 1_{[0, t]} \cdot S\right)_{T}\right)=\sup _{\theta \in \mathcal{A}} E U\left(x+(\theta \cdot S)_{t}\right) .
$$

Definition 3.2. A strategy $\theta^{*} \in \mathcal{A}$ is called always optimal, if for all $t \in[0, T]$ and $x>0$

$$
E U\left(x+\left(\theta^{*} \cdot S\right)_{t}\right)=u_{t}(x) .
$$


We will now analyze to which extent always optimal strategies exist.

Consider at first the case where the drift $\alpha$ is equal to 0 . In this case the price process $S$ is a $P$-local martingale and intuitively one would expect that a risk averse trader will not trade at all. Theorem 3.1 confirms that the maximal expected utility is the utility of the initial capital $U(x)$. Hence in this case the trivial strategy $\theta=0$ is always optimal, whatever the utility function $U$ looks like.

If the drift $\alpha$ is not trivial, however, the situation is different. It turns out that in general always optimal strategies exist only for logarithmic utility functions. Before proving this we define

$$
\bar{Z}_{T}=\sup _{0 \leqslant t \leqslant T} Z_{t}
$$

and

$$
\underline{Z}_{T}=\inf _{0 \leqslant t \leqslant T} Z_{t}
$$

We will only consider the case where

$$
\text { ess inf } \underline{Z}_{T}=0 \text { and } \quad \text { ess sup } \bar{Z}_{T}=\infty \text {. }
$$

Theorem 3.3. Assume that $I=\left(U^{\prime}\right)^{-1}$ is twice continuously differentiable on $(0, \infty)$ and that the conditions $(13)$, (14) and (15) are satisfied. Then an always optimal strategy exists if and only if $U$ is the logarithm up to affine transformations, i.e.

$$
U(x)=a \log (x)+b
$$

for some constants $a>0$ and $b \in \mathbb{R}$.

Proof. Suppose at first that $U(x)=\log (x)$. By Theorem 3.1 we have for any $t \in[0, T]$

$$
\begin{aligned}
u_{t}(x) & =E U\left(I\left(y Z_{t}\right)\right)=E U\left(\frac{1}{y Z_{t}}\right) \\
& =E \log \left(x Z_{t}^{-1}\right)+c=E \log \left[x \mathcal{E}(\alpha \cdot S)_{t}\right]+c=E \log \left[x+(\alpha \mathcal{E}(\alpha \cdot S) \cdot S)_{t}\right]+c .
\end{aligned}
$$

This shows that $\theta^{*}=\alpha \mathcal{E}(\alpha \cdot S)$ is always optimal.

We now prove the converse statement. Let $\theta^{*}$ be an always optimal strategy. By Theorem 3.1 the process

$$
x+\left(\theta^{*} \cdot S\right)=I(y Z)
$$

is a $Q$-martingale. Hence

$$
Z I(y Z)
$$

is a $P$-martingale. Since the function $\phi:(0, \infty) \rightarrow \mathbb{R}, \phi(x)=x I(y x)$ is twice continuously differentiable, we may apply Itô's formula and obtain for $t \in[0, T]$

$$
Z_{t} I\left(y Z_{t}\right)=\phi\left(Z_{t}\right)=\phi(1)+\int_{0}^{t} \phi^{\prime}\left(Z_{s}\right) \mathrm{d} Z_{s}+\frac{1}{2} \int_{0}^{t} \phi^{\prime \prime}\left(Z_{s}\right) \mathrm{d}\langle Z, Z\rangle_{s} .
$$

From this equation we can deduce that the continuous process of bounded variation

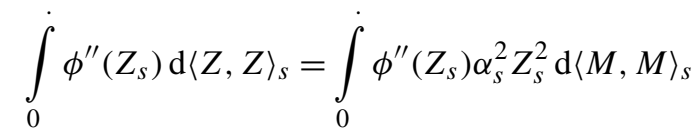


is a local $P$-martingale and hence vanishes. We will now show that $\phi^{\prime \prime}(z)=0$ for all $z>0$. Suppose that this is not true. Then there exist $0<p<q$ such that $\phi^{\prime \prime}$ does not vanish on the interval $(p, q)$. Eq. (15) implies that on the set

$$
A=\left\{(t, \omega): Z_{t}(\omega) \in(p, q)\right\}
$$

we have

$$
\alpha=0 \quad P_{M} \text {-a.s. }
$$

This means that the process $\int_{0}^{\cdot} \alpha^{2} \mathrm{~d}\langle M, M\rangle$ is constant on $A$. Hence also the processes $\int_{0}^{\cdot} \alpha \mathrm{d} M$ and $Z=\mathcal{E}(\alpha \cdot M)$ are constant on $A$ (see [28]), i.e.

$$
1_{A}(t, \omega) Z_{t}(\omega) \text { is constant a.s. }
$$

In other words, the trajectories $t \mapsto Z_{t}(w)$ are a.s. constant on $(p, q)$.

Suppose first that $q<1$ or $p>1$. Since $Z_{0}=1$, it follows that the entire trajectories of $Z$ are above $q$ or below $p$, respectively. This contradicts (15).

Suppose next that $p<1<q$. Since $Z$ is constant on $(p, q)$, we must have $Z=1$, which also contradicts property (15).

Thus we have shown $\phi^{\prime \prime}=0$.

On the other hand we know that

$$
\phi^{\prime}(x)=I(y x)+y x I^{\prime}(y x)
$$

and

$$
\phi^{\prime \prime}(x)=2 y I^{\prime}(y x)+x y^{2} I^{\prime \prime}(y x) .
$$

Hence $I^{\prime}$ solves the differential equation

$$
2 I^{\prime}(z)=-z I^{\prime \prime}(z), \quad z>0 .
$$

By assumption (13) the function $I^{\prime}:(0, \infty) \rightarrow(-\infty, 0)$ satisfies

$$
\lim _{z \rightarrow 0^{+}} I^{\prime}(z)=-\infty
$$

Hence

$$
I^{\prime}(z)=-\frac{a}{z^{2}}
$$

and

$$
I(z)=\frac{a}{z}+c_{1}
$$

for some constants $a>0$ and $c_{1} \in \mathbb{R}$. It follows

$$
U^{\prime}(x)=\frac{a}{x-c_{1}}
$$

and

$$
U(x)=a \log \left(x-c_{1}\right)+c_{2}
$$

for some $c_{2} \in \mathbb{R}$. Note that $c_{1}=0$, because $\lim _{x \rightarrow 0^{+}} U(x)=-\infty$. This completes the proof. 


\subsection{The additional expected utility of an insider}

Let $\mathcal{F}$ and $\mathcal{G}$ be filtrations such that $S$ is complete with respect to both filtrations. We write

$$
S=M+\alpha \cdot\langle M, M\rangle
$$

for the semimartingale decomposition with respect to $\mathcal{F}$ and

$$
S=N+\beta \cdot\langle N, N\rangle
$$

for the decomposition with respect to $\mathcal{G}$. Furthermore we denote by $Q$ the ELMM with respect to $\mathcal{F}$ and by $Q^{\prime}$ the ELMM with respect to $\mathcal{G}$. Notice that

$$
\left.\frac{\mathrm{d} Q}{\mathrm{~d} P}\right|_{\mathcal{F}_{T}}=\mathcal{E}(-\alpha \cdot M)_{T}
$$

and

$$
\left.\frac{\mathrm{d} Q^{\prime}}{\mathrm{d} P}\right|_{\mathcal{G}_{T}}=\mathcal{E}(-\beta \cdot N)_{T} .
$$

Consider now the case where $\mathcal{F}$ is contained in $\mathcal{G}$, i.e. $\mathcal{F}_{t} \subset \mathcal{G}_{t}$ for all $0 \leqslant t \leqslant T$. The following lemma observes that the two ELMMs agree on the smaller world.

Lemma 3.4. On $\mathcal{F}_{T}$ the measures $Q$ and $Q^{\prime}$ are equal, i.e. $\left.Q^{\prime}\right|_{\mathcal{F}_{T}}=\left.Q\right|_{\mathcal{F}_{T}}$. In particular we have

$$
E^{P}\left[\mathcal{E}(-\beta \cdot N)_{T} \mid \mathcal{F}_{T}\right]=\mathcal{E}(-\alpha \cdot M)_{T}
$$

Proof. On the one hand, $S$ is a $\left(Q^{\prime}, \mathcal{G}\right)$-local martingale. Since on the other hand $S$ is adapted to $\mathcal{F}$, it is also a $\left(Q^{\prime}, \mathcal{F}\right)$-local martingale. Completeness of the market implies that the ELMM on $\mathcal{F}$ is unique. Hence $Q^{\prime}$ coincides with $Q$ on $\mathcal{F}_{T}$.

By applying Theorem 3.1 we obtain the following expression for the utility increment

$$
\begin{aligned}
u(\mathcal{G}, x)-u(\mathcal{F}, x) & =E U\left(I\left(y \frac{\mathrm{d} Q^{\prime}}{\mathrm{d} P}\right)\right)-E U\left(I\left(y \frac{\mathrm{d} Q}{\mathrm{~d} P}\right)\right) \\
& =E U\left(I\left(y \mathcal{E}(-\beta \cdot N)_{T}\right)\right)-E U\left(I\left(y \mathcal{E}(-\alpha \cdot M)_{T}\right)\right) .
\end{aligned}
$$

Again we want to express the additional expected utility by means of the information drift $\mu$.

Recall the representation

$$
M=N-\int_{0}^{j_{0}}(\alpha-\beta) \mathrm{d}\langle M, M\rangle \quad \text { a.s. }
$$

with $\mu=\alpha-\beta$ as information drift. Note that for $t \in[0, T]$

$$
\begin{aligned}
\mathcal{E}(-\beta \cdot N)_{t} & =\exp \left[-\int_{0}^{t} \beta \mathrm{d} N-\frac{1}{2} \int_{0}^{t} \beta^{2} \mathrm{~d}\langle M, M\rangle\right] \\
& =\exp \left[\int_{0}^{t} \mu \mathrm{d} N-\int_{0}^{t} \alpha \mathrm{d} M+\int_{0}^{t} \alpha(\beta-\alpha) \mathrm{d}\langle M, M\rangle-\frac{1}{2} \int_{0}^{t} \beta^{2} \mathrm{~d}\langle M, M\rangle\right] \\
& =\mathcal{E}(-\alpha \cdot M)_{t} \mathcal{E}(\mu \cdot N)_{t},
\end{aligned}
$$


which implies

$$
\left.\frac{\mathrm{d} Q^{\prime}}{\mathrm{d} P}\right|_{\mathcal{G}_{T}}=\left.\frac{\mathrm{d} Q}{\mathrm{~d} P}\right|_{\mathcal{F}_{T}} \mathcal{E}(\mu \cdot N)_{T} .
$$

Conditioning on $\mathcal{F}_{T}$ leads to

$$
\left.\frac{\mathrm{d} Q^{\prime}}{\mathrm{d} P}\right|_{\mathcal{F}_{T}}=\left.\frac{\mathrm{d} Q}{\mathrm{~d} P}\right|_{\mathcal{F}_{T}} E\left[\mathcal{E}(\mu \cdot N)_{T} \mid \mathcal{F}_{T}\right],
$$

and by Lemma 3.4 we get

$$
E\left[\mathcal{E}(\mu \cdot N)_{T} \mid \mathcal{F}_{T}\right]=\left.\frac{\mathrm{d} Q^{\prime}}{\mathrm{d} Q}\right|_{\mathcal{F}_{T}}=1 .
$$

We may summarize our findings on the expected additional utility in the following proposition.

Proposition 3.5. The additional expected utility of the insider is equal to

$$
u(\mathcal{G}, x)-u(\mathcal{F}, x)=E\left[U\left(I\left(y \mathcal{E}(-\alpha \cdot M)_{T} \mathcal{E}(-\mu \cdot N)_{T}\right)\right)-U\left(I\left(y \mathcal{E}(-\alpha \cdot M)_{T}\right)\right)\right]
$$

By definition, the insider's expected utility must exceed the regular trader's. In case $U \circ I$ is convex, which is the case for the exponential, power, and logarithmic utility functions for example, but in general does not hold true, the projection result of Lemma 3.4 gives us a direct argument to show this starting with the representation obtained in the preceding proposition. Since $U \circ I$ is convex, Jensen's inequality and equation (16) yield

$$
\begin{aligned}
u(\mathcal{G}, x)-u(\mathcal{F}, x) & =E\left[U\left(I\left(y \frac{\mathrm{d} Q^{\prime}}{\mathrm{d} P}\right)\right)\right]-E\left[U\left(I\left(y \frac{\mathrm{d} Q}{\mathrm{~d} P}\right)\right)\right] \\
& \geqslant E\left[U\left(I\left(y E\left[\mathcal{E}(-\alpha \cdot M)_{T} \mathcal{E}(-\mu \cdot N)_{T} \mid \mathcal{F}_{T}\right]\right)\right)\right]-E\left[U\left(I\left(y \mathcal{E}(-\alpha \cdot M)_{T}\right)\right)\right] \\
& =E\left[U\left(I\left(y \mathcal{E}(-\alpha \cdot M)_{T} E\left[\mathcal{E}(-\mu \cdot N)_{T} \mid \mathcal{F}_{T}\right]\right)\right)\right]-E\left[U\left(I\left(y \mathcal{E}(-\alpha \cdot M)_{T}\right)\right)\right] \\
& =E\left[U\left(I\left(y \mathcal{E}(-\alpha \cdot M)_{T}\right)\right)\right]-E\left[U\left(I\left(y \mathcal{E}(-\alpha \cdot M)_{T}\right)\right)\right]=0 .
\end{aligned}
$$

Remark. We conclude that in general, the utility increment depends - besides the information drift - on the initial wealth and on the intrinsic drift $\alpha$. This is not the case for logarithmic utility functions, where it only depends on the information drift.

\section{Acknowledgements}

We thank an anonymous referee for a very careful and thorough reading of our manuscript and many constructive and helpful remarks.

\section{References}

[1] J. Amendinger, Initial enlargement of filtrations and additional information in financial markets, Thesis, TU Berlin, 1999.

[2] J. Amendinger, D. Becherer, M. Schweizer, A monetary value for initial information in portfolio optimization, Finance and Stochastics 7 (1) (2003) 29-46.

[3] J. Amendinger, P. Imkeller, M. Schweizer, Additional logarithmic utility of an insider, Stochastic Process. Appl. 75 (1998) $263-286$.

[4] S. Ankirchner, Information and semimartingales, PhD thesis, HU Berlin, 2005.

[5] F. Baudoin, Conditioned stochastic differential equations: theory, examples, and applications to finance, Stochastic Process. Appl. 100 (2002) 109-145. 
[6] F. Baudoin, Modeling Anticipations on Financial Markets, Paris-Princeton Lectures on Mathematical Finance 2002, Springer, Berlin, 2003.

[7] F. Baudoin, L. Nguyen-Ngoc, The financial value of a weak information on a financial market, Finance and Stochastics 8 (2004) $415-435$.

[8] F. Biagini, B. Oksendal, A general stochastic calculus approach to insider trading, Preprint, Univ. of Oslo, 2003.

[9] M. Chaleyat-Maurel, T. Jeulin, Grossissement Gaussien de la filtration Brownienne, in: T. Jeulin, M. Yor (Eds.), Grossissements de filtrations : exemples et applications, in : Lecture Notes in Math., vol. 1118, Springer, Berlin, 1985.

[10] J.M. Corcuera, P. Imkeller, A. Kohatsu-Higa, D. Nualart, Additional utility of insiders with imperfect dynamical information, Finance and Stochastics 8 (2004) 437-450.

[11] F. Delbaen, W. Schachermayer, A general version of the fundamental theorem of asset pricing, Math. Ann. 300 (1994) $463-520$.

[12] F. Delbaen, W. Schachermayer, The existence of absolutely continuous local martingale measures, Ann. Appl. Probab. 5 (1995) $926-945$.

[13] F. Delbaen, W. Schachermayer, Arbitrage possibilities in Bessel processes and their relation to local martingales, Probab. Theory Related Fields 102 (1995) 357-366.

[14] F. Delbaen, W. Schachermayer, The variance-optimal martingale measure for continuous processes, Bernoulli 2 (1996) 81-105.

[15] C. Dellacherie, B. Maisonneuve, P.-A. Meyer, Probabilités et potentiel, Chap. XVII-XXIV, Hermann, Paris, 1992.

[16] A. Grorud, M. Pontier, Insider trading in a continuous time market model, Int. J. Theoret. Appl. Finance 1 (1998) $331-347$.

[17] P. Imkeller, Random times at which insiders can have free lunches, Stochastics Stochastics Rep. 74 (2002) 465-487.

[18] P. Imkeller, Malliavin's calculus in insider models: additional utility and free lunches, Math. Finance 13 (2003) $153-169$.

[19] P. Imkeller, M. Pontier, F. Weisz, Free lunch and arbitrage possibilities in a financial market model with an insider, Stochastic Process. Appl. 92 (2001) 103-130.

[20] J. Jacod, Grossissement initial, hypothèse $\left(\mathrm{H}^{\prime}\right)$ et théorème de Girsanov, in: T. Jeulin, M. Yor (Eds.), Grossissements de filtrations : exemples et applications, in : Lecture Notes in Math., vol. 1118, Springer, Berlin, 1985.

[21] T. Jeulin, Semi-martingales et grossissement de filtration, Lecture Notes in Math., vol. 833, Springer, Berlin, 1980.

[22] I. Karatzas, I. Pikovsky, Anticipative portfolio optimization, Adv. Appl. Probab. 28 (1996) 1095-1122.

[23] D. Kramkov, W. Schachermayer, The asymptotic elasticity of utility functions and optimal investment in incomplete markets, Ann. Appl. Probab. 9 (1999) 904-950.

[24] J.A. Leon, R. Navarro, D. Nualart, An anticipative calculus approach to the utility maximization of an insider, Math. Finance 13 (2003).

[25] P.A. Meyer, Sur un théorème de Jacod, Sém. de Probabilités XII, Lecture Notes in Math., vol. 649, Springer, Berlin, 1978.

[26] D. Nualart, The Malliavin Calculus and Related Topics, Springer, Berlin, 1995.

[27] P. Protter, Stochastic Integration and Differential Equations, second ed., Springer, Berlin, 2004.

[28] D. Revuz, M. Yor, Continuous Martingales and Brownian Motion, third ed., Springer, Berlin, 1999.

[29] F. Russo, P. Vallois, Forward, backward and symmetric stochastic integration, Probab. Theory Related Fields 97 (1993) $403-421$.

[30] M. Schweizer, On the minimal martingale measure and the Föllmer-Schweizer decomposition, Stochastic Anal. Appl. 13 (1995) 573-599.

[31] S.-Q. Song, Grossissements de filtrations et problemes connexes, Thèse de doctorat, Univ. Pierre et Marie Curie Paris 6, 1987.

[32] C.T. Wu, Construction of Brownian motions in enlarged filtrations and their role in mathematical models of insider trading, Dissertation, HU Berlin, 1999.

[33] M. Yor, Grossissement de filtrations et absolue continuité de noyaux, in : T. Jeulin, M. Yor (Eds.), Grossissements de filtrations : exemples et applications, in : Lecture Notes in Math., vol. 1118, Springer, Berlin, 1985.

[34] M. Yor, Entropie d'une partition, et grossissement initial d'une filtration, in: T. Jeulin, M. Yor (Eds.), Grossissements de filtrations : exemples et applications, in : Lecture Notes in Math., vol. 1118, Springer, Berlin, 1985.

[35] M. Yor, Inégalités de martingales continues arrêtées à un temps quelconque, I : théorèmes généraux, in : T. Jeulin, M. Yor (Eds.), Grossissements de filtrations : exemples et applications, in : Lecture Notes in Math., vol. 1118, Springer, Berlin, 1985.

[36] M. Yor, Inégalités de martingales continues arrêtées à un temps quelconque, II : le rôle de certains espaces BMO, in : T. Jeulin, M. Yor (Eds.), Grossissements de filtrations : exemples et applications, in : Lecture Notes in Math., vol. 1118, Springer, Berlin, 1985. 